\title{
A multigene family encoding surface glycoproteins in Trypanosoma congolense
}

\author{
Magali Thonnus ${ }^{1}$, Amandine Guérin ${ }^{1,2}$ and Loïc Rivière ${ }^{1, *}$ \\ ${ }^{1}$ Fundamental Microbiology and Pathogenicity Unit, CNRS UMR 5234, Bordeaux University, France. \\ ${ }^{2}$ Current affiliation: CNRS UMR 5235, Montpellier 2 University, France. \\ * Corresponding Author: \\ Dr Loïc Rivière, Fundamental Microbiology and Pathogenicity Unit, CNRS UMR 5234, Bat 3A 1er étage, Bordeaux University; 33000 \\ Bordeaux, France; Tel: +33 557574839; Fax: +33 557574803; E-mail: loic.riviere@u-bordeaux.fr
}

\begin{abstract}
Trypanosoma congolense, the causative agent of the most important livestock disease in Africa, expresses specific surface proteins involved in its parasitic lifestyle. Unfortunately, the complete repertoire of such molecules is far from being deciphered. As these membrane components are exposed to the host environment, they could be used as therapeutic or diagnostic targets. By mining the $T$. congolense genome database, we identified a novel family of lectin-like glycoproteins (TcoClecs). These molecules are predicted to have a transmembrane domain, a tandem repeat amino acid motif, a signal peptide and a C-type lectin-like domain (CTLD). This paper depicts several experimental arguments in favor of a surface localization in bloodstream forms of $T$. congolense. A TcoClec gene was heterologously expressed in U-2 OS cells and the product could be partially found at the plasma membrane. TcoClecs were also localized at the surface of $T$. congolense bloodstream forms. The signal was suppressed when the cells were treated with a detergent to remove the plasma membrane or with trypsin to "shave" the parasites and remove their external proteins. This suggests that TcoClecs could be potential diagnostic or therapeutic antigens of African animal trypanosomiasis. The potential role of these proteins in $T$. congolense as well as in other trypanosomatids is discussed.
\end{abstract}

doi: 10.15698/mic2017.03.562 Received originally: 20.10.2016; in revised form: 08.02.2017, Accepted 13.02.2017, Published 02.03.2017.

Keywords: Trypanosoma congolense, surface glycoprotein, multigene family, lectin-like, trypanosomes.
Abbreviations:
BSF - bloodstream forms, CTLD - C-type lectin-like domain, EMF - epimastigote forms, IFA - immunofluorescence assay, MCF - metacyclic forms, PCF - procyclic forms, VSG - variant surface glycoprotein.

\section{INTRODUCTION}

Trypanosomes are eukaryotic microbes found in different parts of the world. In Africa, these deadly parasites are responsible for neglected diseases called sleeping sickness in human (Trypanosoma brucei gambiense and Trypanosoma brucei rhodesiense) [1] and nagana in cattle and game animals (Trypanosoma brucei brucei, Trypanosoma congolense and Trypanosoma vivax) [2, 3].

The development of trypanosomes follows a complex lifecycle. Bloodstream forms (BSF) proliferate in the blood of the infected mammalian host and are ingested by an insect (called tsetse fly, Glossina spp) during the meal. Then, they differentiate into procyclic forms (PCF) in the midgut and migrate to the salivary glands and proboscis where they attach as epimastigote forms (EMF). Finally, they differentiate into infective metacyclic forms (MCF) that are transmitted to a new mammalian host during the next blood meal.

Trypanosomes have become an interesting model to study biological processes. For example, they possess gly- cosomes, which are specialized peroxysomes involved in glycolysis, a unique tubular mitochondrion, and a flagellar pocket that is the only site for endo- and exocytosis [4, 5]. Moreover, RNA editing, glycophosphatidylinositol (GPI) anchoring, trans-splicing and antigenic variation are biological phenomena that were initially discovered in these parasites [6-8].

T. b. brucei is widely used as a model organism in African trypanosome biology. On the contrary, T. congolense, the main causative agent of African animal trypanosomiasis, is poorly studied.

These two species have different behavior upon in vivo infection and in vitro cultivation. In the mammalian host, $T$. congolense adheres to endothelial cells and red blood cells, whereas T. b. brucei does not [9]. Interestingly, in vitro $T$. congolense BSF adhere directly to the flask but not $T$. $b$. brucei. This could reflect a different composition or property of the plasma membrane. Actually, in both species, the entire surface is covered by millions of copies of a single variant surface glycoprotein (VSG) which constitute a 
A

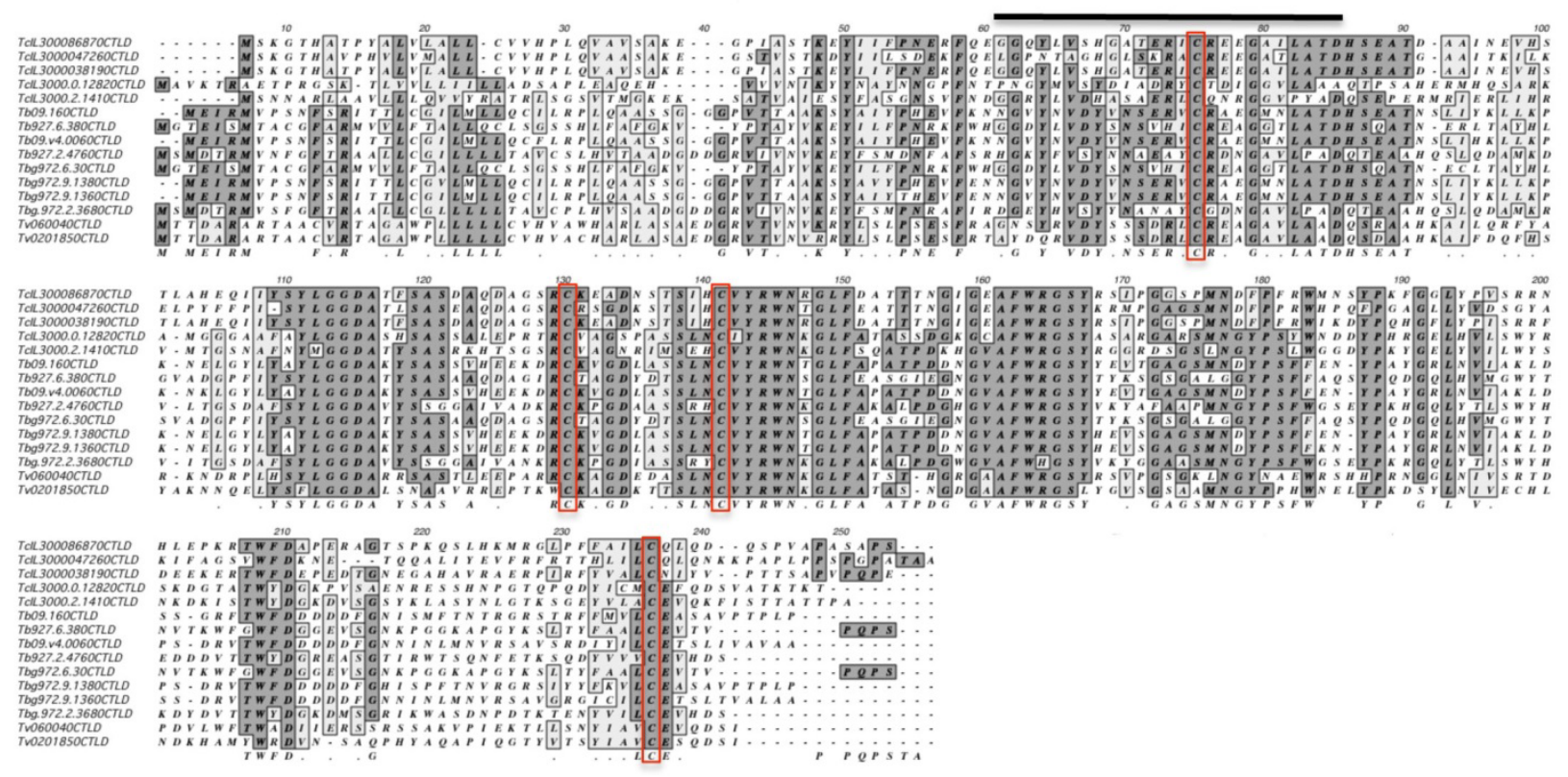

B

Trypanosoma congolense

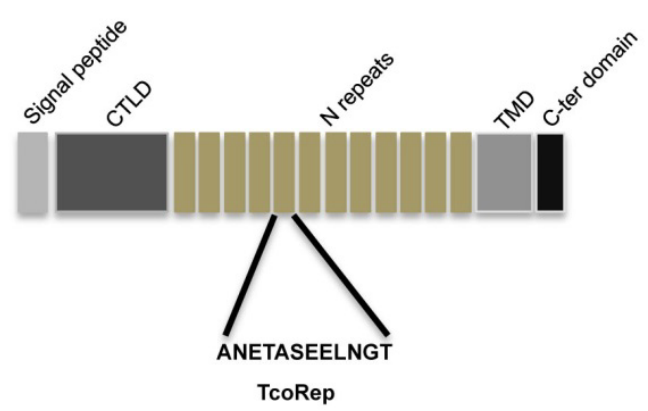

Trypanosoma brucei spp

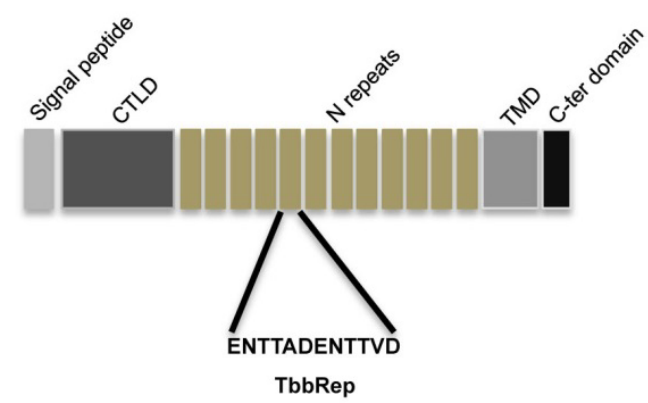

FIGURE 1: Comparison of Clec proteins in African trypanosomes. (A) Alignment of C-type lectin-like domain (CTLD) from African trypanosomes Clec proteins. Sequences were extracted and aligned using MacVector V11. Putative critical cysteines important for protein folding are indicated (red boxes) as well as link module (black line). Dark grey boxes contain identical residues, light grey boxes contain conservative changes. TcIL3000, Trypanosoma congolense IL3000 (reference strain); Tb, Trypanosoma brucei brucei; Tbg, Trypanosoma brucei gambiense; Tv, Trypanosoma vivax. (B) General features shared by Clec proteins in T. congolense and T. b. brucei. TMD, transmembrane domain.

" coat " that masks other antigens and fools the immune system of the host $[10,11]$. Nevertheless, VSG are not responsible for adhesion and the repertoire of surface proteins is not completely well-known. Our study aimed to discover such new molecules in T. congolense BSF.

As lectins are exposed on the surface of cells, we performed a search in the African trypanosomes genome database in order to identify candidates. We found a family of proteins that possesses several features of surface molecules, i.e. a transmembrane domain, a signal peptide, a tandem repeat amino acid motif and a C-type lectin fold domain $[12,13]$. We called these proteins TcoClecs. Interestingly, these molecules have already been detected in the cell-surface phylome [14], and a recent study has shown that TcoClec orthologs in T. b. brucei are glycoproteins retained in the endoplasmic reticulum (ER) [15].

Here we report that TcoClecs are exposed on the surface of $T$. congolense BSF.

\section{RESULTS}

In silico identification of new putative $T$. congolense lectins

Our first goal was to identify in silico new genes that could code for surface proteins of $T$. congolense BSF. In order to minimize the number of potential candidates, we focused our search on lectins. We chose the C-type lectin-like domain (CTLD, InterPro IPR016187) because in metazoan parasites, lectins that contain this motif are involved in 
A

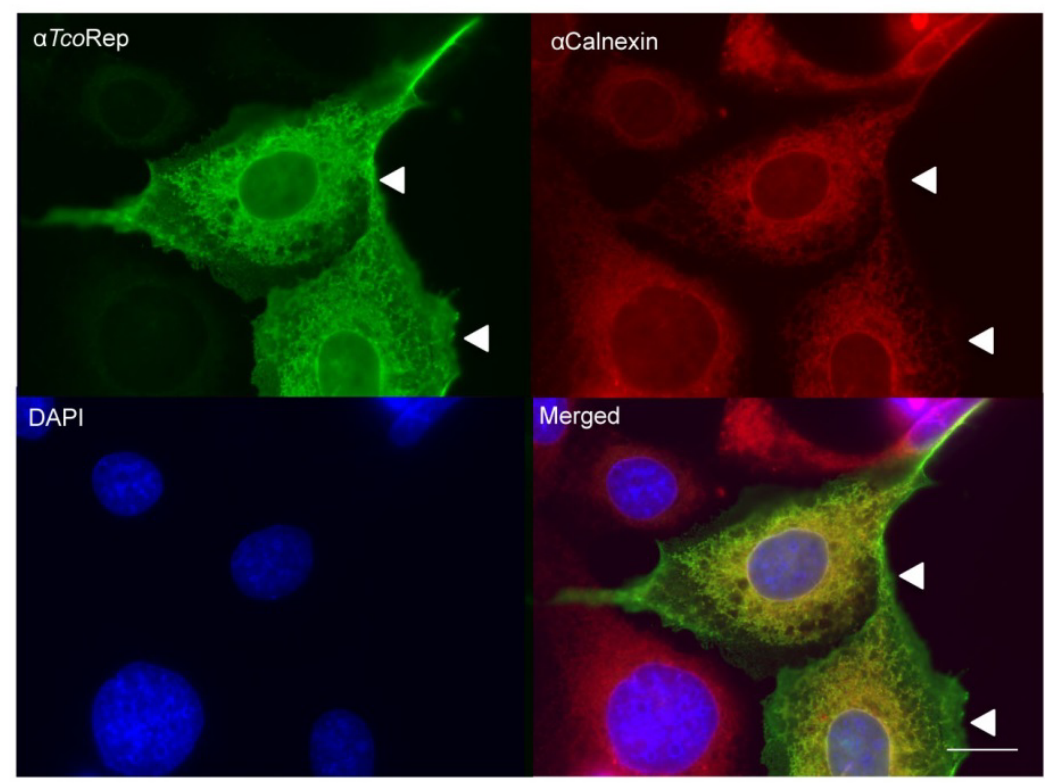

FIGURE 2: Immunofluorescence analysis of U-2 OS cells expressing a TcoClec protein. U-2 OS cells expressing a TcoClec protein are indicated (white arrowheads). (A) Cells were stained with anti-TcoRep, anticalnexin and DAPI. Merged picture (bottom right) shows that $\mathrm{TcoClec}$ and calnexin colocalize partially. (B) Three patterns could be observed in the same proportions: endoplasmic reticulum (ER, left), plasma membrane (PM, middle) and both localizations (right). Cells were stained with anti-TcoRep and DAPI. Bar, $20 \mu \mathrm{m}$.
B

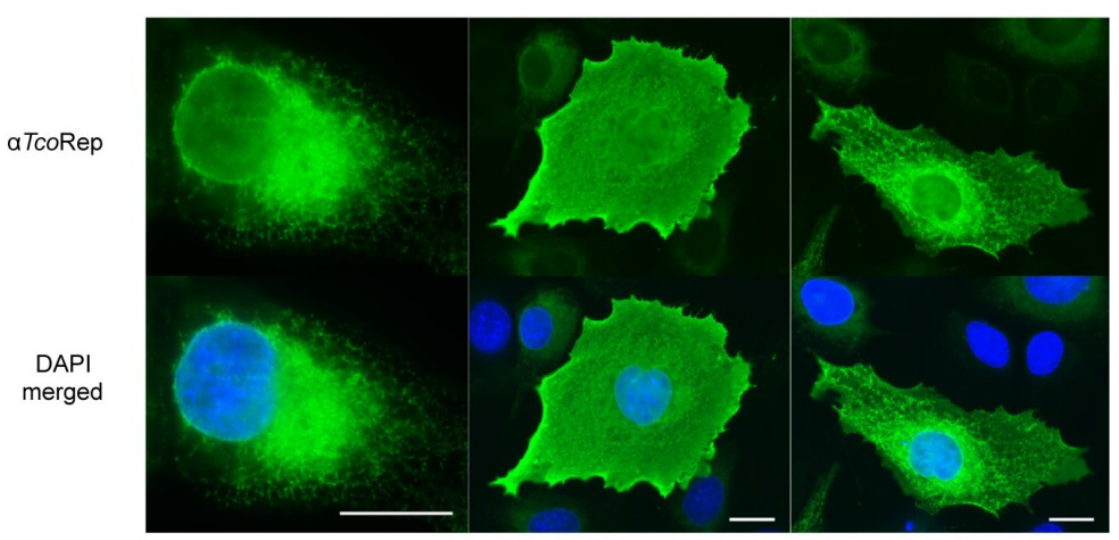

host-parasite interaction [16]. In protozoans, this domain was only described in the human pathogen Cryptosporidium parvum [17]. We restricted our search to multigene families of proteins with a transmembrane domain and a signal peptide. These last features are common to many unicellular surface proteins [18]. By mining the $T$. congolense genome with the Tritryp website (Tritrypdb.org), we found genes (see the materials and methods section) corresponding to a unique family. Interestingly, this family was already identified in the cell-surface phylome as «Fam77 " "Lectin-like membrane protein " [14]. Also, orthologs in $T$. b. brucei have been described recently and are called TbIGP (invariant glycoproteins) [15]. This family could be divided in subfamilies according to phylogenetic analysis [15]. Alignment of African trypanosomes CTLDs revealed both conserved and variable regions. Four cysteine residues are conserved and could be essential for correct folding. In addition, a link module important for carbohydrate recognition is present (Figure 1A) [17, 19].

Strikingly, these proteins have a tandem repeat amino acid motif. The sequence of this motif is different between
T. congolense and T. b. brucei (Figure 1B). We decided to name these proteins TcoClecs according to current nomenclature $[13,17]$.

\section{TcoClecs can be heterologously expressed on the surface of U-2 OS cells}

We employed polyclonal antibodies directed against the amino acid motif (anti TcoRep, Figure 1B) to characterize further these molecules. As protein expression in heterologous cells can help to decipher localizations [20], we used this strategy to first prove the specificity of our antibodies. U-2 OS cells do not possess any TcoClec orthologs and are well-suited for heterologous expression of trypanosomal proteins [21-23]. From Figure 2, it can be seen that our antibodies react only with transfected cells, whereas the control marker (calnexin) is detected in all cells. Interestingly, TcoClec partially colocalizes with calnexin, suggesting that the protein could be distributed in the ER. In addition, some signal is seen on the edge of the transfected cells. This could correspond to a plasma membrane localization (Figure 2A). Moreover, three localization patterns are ob- 
A

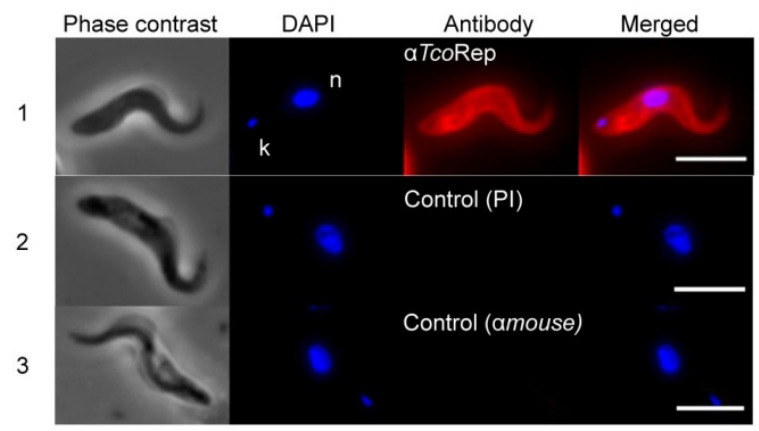

C

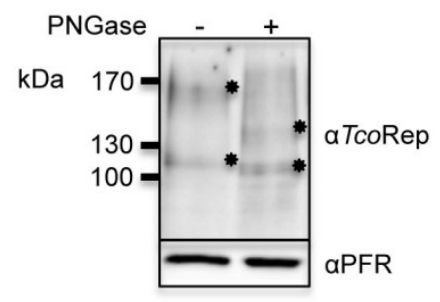

D

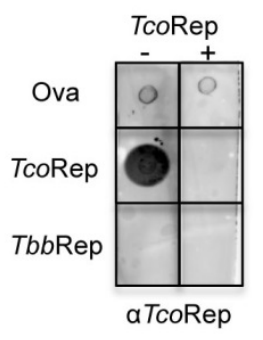

\section{B}

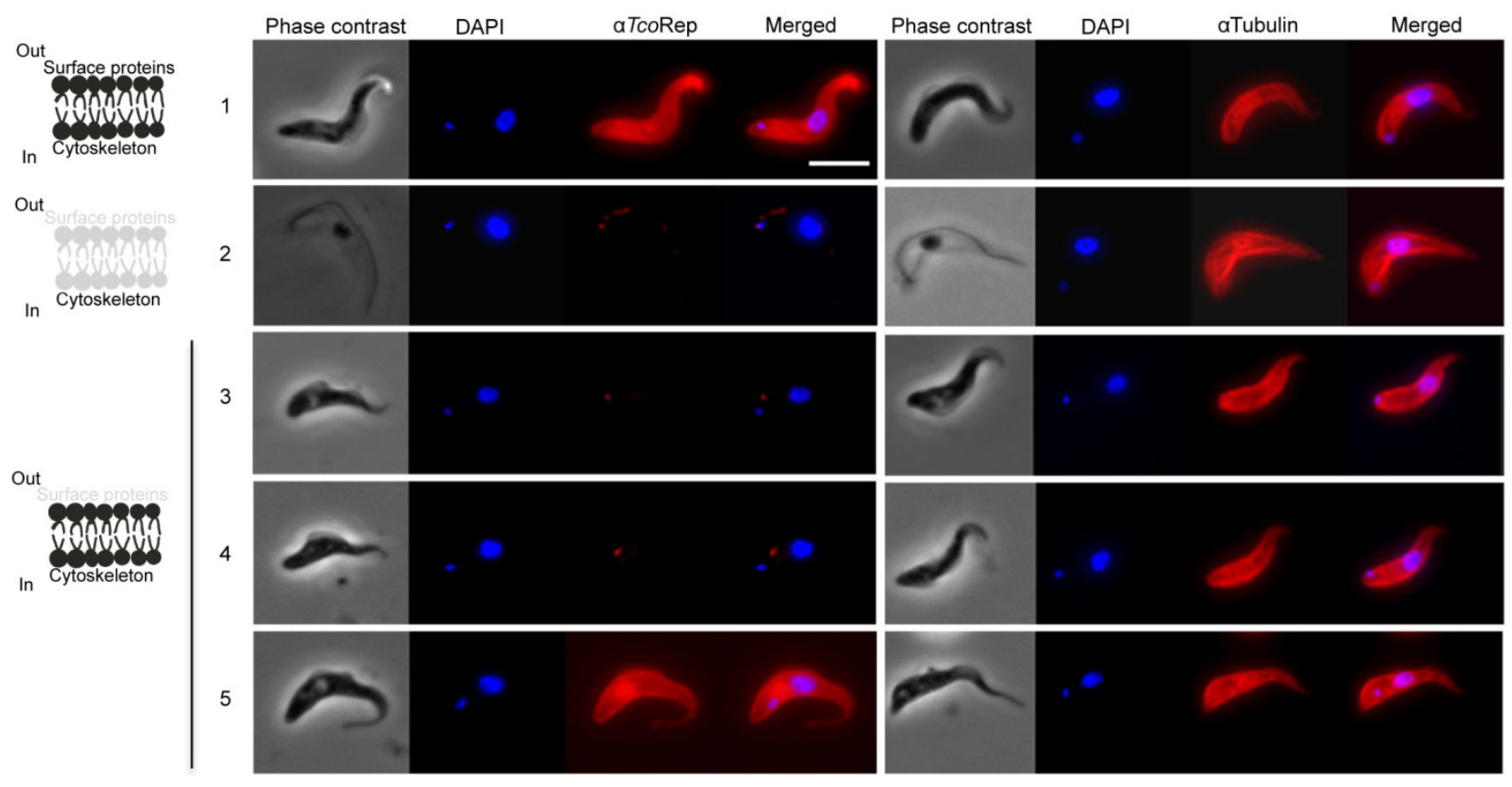

FIGURE 3: Expression and cellular localization of TcoClec in BSF of $T$. congolense. (A) Immunofluorescence analysis of $T$. congolense BSF. Cells were stained with mouse anti-TcoRep (1); control pre-immune serum (2); or secondary antibody alone (3). DAPI was used to stain both nucleus ( $\mathrm{n}$ ) and kinetoplast (k). Phase contrast images are shown on the left. (B) Immunofluorescence analysis. Images are typical examples showing phase contrast, DAPI staining of the nucleus and the kinetoplast, and staining with anti-TcoRep or anti-tubulin. Whole-cell T. congolense BSF (1); cytoskeleton-extracted cells (treated with NP40) (2); and trypsin-treated cells (" shaved " parasites) with IFA performed immediately (3), $5 \mathrm{~h} \mathrm{(4)}$ and $14 \mathrm{~h} \mathrm{(5)}$ after the treatment. A schematic illustration is shown on the left to explain the effects of the treatments on Tco BSF. Bar, $5 \mu \mathrm{m}$. (C) Western blot analysis. Tco BSF cell lysates treated (+) or not (-) with PNGase F. Mouse anti-TcoRep and rabbit anti-PFR (control) were used. Asterisks show band shifts. (D) Peptide competition assay (PCA). Dot blot analysis developed with anti-TcoRep antibodies. Anti-TcoRep antibodies were incubated (+) or not (-) with the TcoRep peptide. Ovalbumin (Ova), TcoRep peptide and TbbRep peptide were spotted on the filters.

served: ER, plasma membrane and both ER and plasma membrane (Figure 2B). These results suggest that in U-2 OS cells, heterologously-expressed TcoClec can be directed to the membrane. Finally, these experiments validate our antibodies as a specific tool for immunofluorescence assay (IFA).
TcoClecs are membrane glycoproteins exposed at the surface of $T$. congolense BSF

Figure $3 \mathrm{~A}$ shows that anti-TcoRep stains the whole cell, with a marked signal at the periphery. It is a typical plasma membrane staining. No signal could be obtained with control antibodies (pre-immune and secondary antibody alone). This result suggests that Tcoclecs could be distributed on the plasma membrane. Unfortunately, there is no $T$. congolense BSF surface marker available to see potential 
colocalization. To overcome these technical difficulties, we decided to perform cell treatments followed by IFA. In all these experiments we used the detection of tubulin to monitor the quality of our preparations. Indeed, tubulin is the main component of the subcellular corset, which is a typical cytoskeleton network found immediately under the plasma membrane [24]. With an anti-tubulin antibody, the signal looks like a membrane staining in IFA (Figure 3B, panel 1 , right). The first set of treatment consisted in removing the plasma membrane with the help of a detergent. This method is currently used to analyze parasite cytoskeletons $[25,26]$. As can be seen from Figure 3B (panels 1 and 2 ), the effect of the detergent (NP40) can be observed on phase contrast images, which constitute a good quality control of the experiment. In these cells, the typical membrane-like staining disappeared completely by using antiTcoRep, whereas tubulin could still be detected properly (Figure 3B, panel 2, left and right). This indicates that the signal observed previously (Figure $3 \mathrm{~A}$, panel 1) could actually correspond to the plasma membrane.

The purpose of the second set of experiment was to remove the external part of membrane proteins. We shaved the parasites by means of a protease treatment. We used trypsin to detach the parasites from the culture flask and presumed that this treatment removed surface proteins [27]. In detached trypsin-treated parasites, the TcoClec signal disappears while that of tubulin is still present (Figure 3B, panel 3). Phase contrast images show that the shape of the cells is not altered by the treatment. After incubation with trypsin, parasites were still alive and could be put back into culture. Interestingly, the TcoClec signal was absent $5 \mathrm{~h}$ later and reappeared $14 \mathrm{~h}$ later. Altogether these results suggest not only that TcoClecs are plasma membrane proteins but also that they are exposed at the cell surface. Finally, as glycosylation sites could be predicted, cell lysates from $T$. congolense BSF were PNGasetreated and analyzed to see if these proteins are truly posttranslationally modified. Figure $3 \mathrm{C}$ shows that bands shift after glycosidase treatment, indicating that TcoClecs are glycosylated. Quality control of anti-TcoRep was done by using dot blot analysis. On the left panels of Figure 3D, we can see that our antibodies recognize specifically the peptide motif of $T$. congolense but not that of $T$. b. brucei. The right panels depict the same experiment where the antiTcoRep was pre-incubated with TcoRep peptide (peptide competition assay). As can be seen in the figure, the signal previously observed disappears, which demonstrates the specificity of our antibody.

\section{DISCUSSION}

In our study, we identified a new family of membrane glycoproteins containing a putative CLTD with members expressed at the surface of $T$. congolense BSF. This is of particular importance because, to our knowledge, only a few surface molecules have been described so far.

Interestingly, this gene family already appeared in the cell-surface phylome published by Jackson et al. [14] and was denominated "lectin-like membrane protein »
(Fam77). Recently, another study focused on this gene family in $T$. b. brucei [15]. The authors provided a very complete and detailed phylogenetic analysis confirming the homogeneity in African trypanosomes. Interestingly, they are localized in the ER in T. b. brucei, whereas they are membrane surface-exposed in $T$. congolense. This could reflect species peculiarities such as tissue distribution or pathogenicity.

In non metazoan organisms, CTLD-containing proteins are involved in adhesion to the host [28]. In African trypanosomes, the molecules implicated in host contact are not well-documented. Moreover, as pathogenicity and parasite behavior are different from one species to another there is no strictly conserved mechanisms that could solve the problematic in all African trypanosomes. This striking fact is exemplified by the differences between $T$. congolense, which adheres to endothelial cells and red blood cells, and T. b. brucei, which does not. Here, proteins involved in host cell adhesion are unknown. It has been hypothesized that candidates should bind to sugars [9], but this has to be experimentally proven. Concerning $T . b$. brucei, it is even more complicated. These parasites circulate freely in blood vessels but can also invade tissues [29-32]. At one point they should first adhere then penetrate [33]. There is a lack of literature concerning the molecules involved in these processes. Moreover, the expression and localization of the proteins involved in these events should be highly controlled. This can also be the case in the insect vector, where the journey from the gut to the salivary glands is long and necessitates at some point adhesion to the fly tissues. We are currently investigating whether these proteins could be implicated in adhesion, and the T. congolense model is well-suited to address that question.

$T$. congolense is the main causative agent of animal trypanosomiasis, arguably the most important livestock disease in Africa, due to its devastating effects on livestock production across sub-Saharan Africa. Important limitations exist concerning diagnosis and treatments [34]. Glycoproteins are antigenic and are used in a number of diagnostic tests. For example, the serodiagnosis of sleeping sickness detects antibodies against a variant surface glycoprotein [35] but does not work with animal trypanosomes. As the TcoClecs described in this paper should face the host's immune system, they could be used as a diagnostic or therapeutic target.

\section{MATERIALS AND METHODS \\ In silico identification of TcoClec genes in $T$. congolense}

First, we mined the $T$. $b$. brucei genome with the Tritryp website (Tritrypdb.org) using " similarity/pattern " and "InterPro domain » corresponding to C-type lectin fold domain. Second, we searched for TMD and signal peptide. Strikingly, all these genes are related, showing primary sequence similarities, and orthologs are found only in African trypanosomes including $T$. congolense. These genes belong to the Fam77 described elsewhere $[14,15]$. 


\section{Cell lines and cell culture}

T. congolense IL3000 BSF (kindly provided by the International Livestock Research Institute, Nairobi, Kenya) were cultured in Eagle's Minimum Essential Medium (Sigma) supplemented with $25 \mathrm{mM}$ sodium bicarbonate, $25 \mathrm{mM}$ Hepes, $5.5 \mathrm{mM}$ glucose, $1 \mathrm{mM}$ pyruvate, $0.04 \mathrm{mM}$ adenosine, $0.1 \mathrm{mM}$ hypoxanthine, $0.02 \mathrm{mM}$ thymidine, $0.02 \mathrm{mM}$ bathocuprone, $200 \mathrm{mM}$ glutamine and $20 \%$ goat serum at $34^{\circ} \mathrm{C}$ in a humidified atmosphere containing $5 \% \mathrm{CO}_{2}$ [36].

U-2 OS cells (human bone osteosarcoma epithelial cells, ATCC ${ }^{\circledR}$ Number: HTB-96, [23]) were grown in D-MEM Glutamax (Gibco) supplemented with $10 \%$ fetal calf serum and $1 \%$ penicillin-streptomycin at $37^{\circ} \mathrm{C}$ plus $5 \% \mathrm{CO}_{2}$.

\section{Plasmid construction and transient transfection of U-2 OS cells}

A TcoClec ORF (TcIL3000.0.50510) was amplified from T. congolense IL 3000 genomic DNA using the two specific primers TcoClec-Fw (5'-CTCGAGATGAGCAAAGGGAAACA-CG-3') and TcoClecSTOP-rev (5'-CTACTGAGCAACCGCCGG-CGAC-3'), cloned into pcDNA3.1/CT-TOPO (Invitrogen) and sequenced. Exponentially growing cells were transfected with $250 \mathrm{ng}$ DNA (TcoClec or control) using Lipofectamine 2000 in OPTIMEM (Invitrogen) according to the manufacturer's instructions and processed for IFA $24 \mathrm{~h}$ post-transfection. To control transfection efficiency, the pcDNA3.1 Bilbo plasmid was used [22]. A transfection without DNA was used as negative control.

\section{Production of anti-TcoRep antibodies}

A peptide corresponding to a sequence found in the repeats $\left(\mathrm{H}_{2} \mathrm{~N}\right.$-CEELNGTDANETASEELNGTDANETAS-CONH ${ }_{2}$; TcoRep peptide) was synthesized and conjugated to ovalbumin. Antiserum was raised in mice by 4 injections at 15-day intervals of $25 \mu \mathrm{g}$ of peptides emulsified with complete (first injection) or incomplete Freund's adjuvant (following boosts).

\section{Removal of surface proteins by trypsin treatment (" shaving experiment ")}

TCIL3000 BSF in suspension were eliminated and adherent parasites were briefly washed twice in $4.5 \mathrm{~mL}$ PBS supplemented with glucose $(1.8 \mathrm{~g} / \mathrm{L}, \mathrm{gPBS})$. Cells were then incubated for $3 \mathrm{~min}$ with $0.5 \mathrm{mg} / \mathrm{mL}$ trypsin solution (SigmaT0134) at RT. To stop the reaction, detached parasites were put at $4^{\circ} \mathrm{C}$, collected and washed in gPBS. Culture medium was added; one part was directly analyzed by IFA, and the other was put back in the incubator. Subsequent IFA were performed with these parasites.

\section{Fluorescence microscopy}

T. congolense BSF

Plastic-adherent trypanosomes grown in culture were scrapped, collected by centrifugation ( $500 \mathrm{~g}, 5 \mathrm{~min}, \mathrm{RT})$ and washed in gPBS.

For whole-cell and " shaving " experiments, trypanosomes were fixed with paraformaldehyde (PFA, 3\%) as described elsewhere [22].

For cytoskeleton-extracted cell experiments, parasites were extracted with Pipes buffer (100 mM Pipes pH 6.9, 1 mM $\mathrm{MgCl}_{2}, 0.05 \% \mathrm{NP} 40$ ) for $3 \mathrm{~min}$, washed and fixed with PFA (3\%).

Slides were incubated with mouse anti-TcoRep (diluted 1:100) or mouse anti-beta tubulin (diluted 1:200) followed by
Alexa Fluor 596-conjugated goat anti-mouse secondary antibody (diluted 1:100) (Invitrogen). The nucleus and the kinetoplast were stained with DAPI $(10 \mu \mathrm{g} / \mathrm{mL})$. Cells were viewed using a Zeiss Axio Imager Z1 fluorescent microscope; images were captured using the MetaMorph ${ }^{\circledR}$ software (Molecular Devices) and processed using ImageJ software.

\section{U-2 OS cells}

Cells grown on coverslips were washed briefly with PBS and fixed in 3\% PFA for 20 min. Cells were neutralized in glycine (0.1 $\mathrm{M}$ in PBS) and treated for immunolabelling as described [37]. Primary antibodies mouse anti-TcoRep (1:250) and anticalnexin (1:400) were incubated for $1 \mathrm{~h}$ in a moist chamber. After three washes, Alexa Fluor 488-conjugated goat antimouse secondary antibody and Alexa Fluor 594-conjugated goat anti-rabbit secondary antibody (diluted 1:100) (Invitrogen) were added for $1 \mathrm{~h}$. The nuclei were stained with DAPI $(10 \mu \mathrm{g} / \mathrm{mL})$ and cells were observed as described above.

\section{$\mathrm{N}$-glycosylation analysis}

Parasites were treated with PNGase F (New England Biolabs). $1 \times 10^{8}$ cells were washed in PBS, resuspended in $2 \%$ SDS and then heated to $100^{\circ} \mathrm{C}$ for $10 \mathrm{~min}$. Samples were treated with PNGase $\mathrm{F}$ for $3 \mathrm{~h}$ at $37^{\circ} \mathrm{C}$ according to the manufacturer's instructions.

\section{Western blot and dot blot analysis}

For Western blot analysis, total protein lysates of $T$. congolense BSF were separated by SDS-PAGE (4-20\% Mini PROTEAN TGX stain-free precast gradient gels, Bio-Rad) and blotted on PVDF filters (Bio-Rad). The membranes were blocked with PBS $5 \%$ milk powder for $1 \mathrm{~h}$ at RT. For dot blot analysis, $200 \mathrm{ng}$ of peptides were spotted directly on the membranes (nitrocellulose $0.45 \mu \mathrm{m}$, Bio-Rad). Primary and secondary antibodies were diluted in TBS (Tris-buffered saline: $10 \mathrm{mM}$ Tris, $150 \mathrm{mM}$ $\mathrm{NaCl}, \mathrm{pH}$ 7.4) with $0.05 \%$ Tween 20 and 5\% BSA powder: mouse anti-TcoRep 1:250; rabbit anti-PFR (paraflagellar rod) 1:5000; anti-mouse conjugated to horseradish peroxidase (KPL) 1:5000; or anti-rabbit conjugated to horseradish peroxidase (KPL) 1:10000. Revelations were done using Clarity Western ECL Substrate (Bio-Rad) according to the manufacturer's instructions.

\section{Peptide competition assay}

Anti-TcoRep was diluted (1:250 in TBS supplemented with Tween 20 and BSA) and equally divided into two tubes; $25 \mu \mathrm{g}$ of TcoRep peptide was added to one tube, and the equivalent of water was added to the other tube. After $1 \mathrm{~h}$ of incubation at RT, the dot blot was pursued as previously described.

\section{Ethics statement}

All animal procedures were carried out in strict accordance with the French legislation (Rural Code articles L 214-1 to L 214-122 and associated penal consequences) and European Union (2010/63/EU) guidelines for the care of laboratory animals and were approved by the Ethical Committee (C2EA-50) of the Centre National de la Recherche Scientifique and by the University of Bordeaux animal care and use committee. All efforts were made to minimize animal suffering. 


\section{ACKNOWLEDGMENTS}

We would like to acknowledge J. Izotte for technical help with immunizations, and V. Coustou, M. Bonhivers, D.R. Robinson and B. Roger for discussions and technical help. This work was supported by the CNRS, the Ministère de I'Education Nationale de la Recherche et de l'Enseignement Supérieur, the University of Bordeaux, the Laboratoire d'Excellence (LabEx) ParaFrap ANR-11-LABX0024 and the Fédération de Recherche TransBioMed.

\section{CONFLICT OF INTEREST}

We declare no conflict of interest.

\section{REFERENCES}

1. Malvy D and Chappuis $F$ (2011). Sleeping sickness. Clin Microbiol Infect 17(7): 986-995.

2. Barrett MP, Boykin DW, Brun R, and Tidwell RR (2007). Human African trypanosomiasis: pharmacological re-engagement with a neglected disease. Br J Pharmacol 152(8): 1155-1171.

3. Van den Bossche $P$ (2001). Some general aspects of the distribution and epidemiology of bovine trypanosomosis in southern Africa. Int J Parasitol 31(5-6): 592-598.

4. Bringaud F, Rivière L, and Coustou V (2006). Energy metabolism of trypanosomatids: adaptation to available carbon sources. Mol Biochem Parasitol 149(1): 1-9.

5. Field MC and Carrington M (2009). The trypanosome flagellar pocket. Nat Rev Microbiol 7(11): 775-786.

6. Benne R, Van den Burg J, Brakenhoff JP, Sloof P, Van Boom JH, and Tromp MC (1986). Major transcript of the frameshifted coxll gene from trypanosome mitochondria contains four nucleotides that are not encoded in the DNA. Cell 46(6): 819-826.

7. Horn D (2014). Antigenic variation in African trypanosomes. Mol Biochem Parasitol 195(2): 123-129.

8. Ngô H, Tschudi C, Gull K, and Ullu E (1998). Double-stranded RNA induces mRNA degradation in Trypanosoma brucei. Proc Natl Acad Sci U S A 95(25): 14687-14692.

9. Hemphill A, Frame I, and Ross CA (1994). The interaction of Trypanosoma congolense with endothelial cells. Parasitology 109 (Pt 5): 631-641. PMID: 7831098.

10. Mugnier MR, Stebbins CE, and Papavasiliou FN (2016). Masters of disguise: antigenic variation and the VSG coat in Trypanosoma brucei. PLoS Pathog 12(9): e1005784.

11. Pays E and Nolan DP (1998). Expression and function of surface proteins in Trypanosoma brucei. Mol Biochem Parasitol 91(1): 3-36.

12. Drickamer K (1999). C-type lectin-like domains. Curr Opin Struct Biol 9(5): 585-590.

13. Zelensky AN and Gready JE (2005). The C-type lectin-like domain superfamily. FEBS J 272(24): 6179-6217.

14. Jackson AP, Allison HC, Barry JD, Field MC, Hertz-Fowler C, and Berriman M (2013). A cell-surface phylome for African trypanosomes. PLoS Negl Trop Dis 7(3): e2121.

15. Allison H, O'Reilly AJ, Sternberg J, and Field MC (2014). An extensive endoplasmic reticulum-localised glycoprotein family in trypanosomatids. Microb Cell 1(10): 325-345.

16. Loukas A and Maizels RM (2000). Helminth C-type lectins and hostparasite interactions. Parasitol Today 16(8): 333-339.

\section{COPYRIGHT}

(C) 2017 Thonnus et al. This is an open-access article released under the terms of the Creative Commons Attribution (CC BY) license, which allows the unrestricted use, distribution, and reproduction in any medium, provided the original author and source are acknowledged.

Please cite this article as: Magali Thonnus, Amandine Guérin and Loïc Rivière (2017). A multigene family encoding surface glycoproteins in Trypanosoma congolense. Microbial Cell 4(3): 90-97. doi: 10.15698/mic2017.03.562

17. Bhalchandra S, Ludington J, Coppens I, and Ward HD (2013). Identification and characterization of Cryptosporidium parvum $\mathrm{Clec}$, a novel C-type lectin domain-containing mucin-like glycoprotein. Infect Immun 81(9): 3356-3365.

18. Scherf A, Lopez-Rubio JJ, and Riviere L (2008). Antigenic variation in Plasmodium falciparum. Annu Rev Microbiol 62: 445-470.

19. Kohda D, Morton CJ, Parkar AA, Hatanaka H, Inagaki FM, Campbell ID, and Day AJ (1996). Solution structure of the link module: a hyaluronan-binding domain involved in extracellular matrix stability and cell migration. Cell 86(5): 767-775.

20. Jackson DG, Smith DK, Luo C, and Elliott JF (1993). Cloning of a novel surface antigen from the insect stages of Trypanosoma brucei by expression in COS cells. J Biol Chem 268(3): 1894-1900.

21. Dacheux D, Landrein N, Thonnus M, Gilbert G, Sahin A, Wodrich H, Robinson DR, and Bonhivers M (2012). A MAP6-related protein is present in protozoa and is involved in flagellum motility. PLoS One $7(2)$ : e31344.

22. Florimond C, Sahin A, Vidilaseris K, Dong G, Landrein N, Dacheux D, Albisetti A, Byard EH, Bonhivers M, and Robinson DR (2015). Correction: BILBO1 is a scaffold protein of the flagellar pocket collar in the pathogen Trypanosoma brucei. PLoS Pathog 11(4): e1004844.

23. Heldin $\mathrm{CH}$, Johnsson A, Wennergren S, Wernstedt C, Betsholtz C, and Westermark B (1986). A human osteosarcoma cell line secretes a growth factor structurally related to a homodimer of PDGF A-chains. Nature 319(6053): 511-514.

24. Gull K (1999). The cytoskeleton of trypanosomatid parasites. Annu Rev Microbiol 53: 629-655.

25. Bonhivers M, Nowacki S, Landrein N, and Robinson DR (2008). Biogenesis of the trypanosome endo-exocytotic organelle is cytoskeleton mediated. PLoS Biol 6(5): e105.

26. Sunter JD, Varga V, Dean S, and Gull K (2015). A dynamic coordination of flagellum and cytoplasmic cytoskeleton assembly specifies cell morphogenesis in trypanosomes. J Cell Sci 128(8): 1580-1594.

27. Olaya-Abril A, Jiménez-Munguía I, Gómez-Gascón L, and Rodríguez-Ortega MJ (2014). Surfomics: shaving live organisms for a fast proteomic identification of surface proteins. J Proteomics 97: 164-176.

28. Hostetter MK (1994). Adhesins and ligands involved in the interaction of Candida spp. with epithelial and endothelial surfaces. Clin Microbiol Rev 7(1): 29-42.

29. Caljon G, Van Reet N, De Trez C, Vermeersch M, Pérez-Morga D, and Van Den Abbeele J (2016). The dermis as a delivery site of Trypanosoma brucei for tsetse flies. PLoS Pathog 12(7): e1005744. 
30. Capewell P, Veitch NJ, Turner CMR, Raper J, Berriman M, Hajduk SL, and MacLeod A (2011). Differences between Trypanosoma brucei gambiense groups 1 and 2 in their resistance to killing by trypanolytic factor 1. PLoS Negl Trop Dis 5(9): e1287.

31. Mogk S, Meiwes A, Shtopel S, Schraermeyer U, Lazarus M, Kubata $B$, Wolburg H, and Duszenko M (2014). Cyclical appearance of African trypanosomes in the cerebrospinal fluid: new insights in how trypanosomes enter the CNS. PLoS One 9(3): e91372.

32. Trindade S, Rijo-Ferreira F, Carvalho T, Pinto-Neves D, Guegan $F$, Aresta-Branco F, Bento F, Young SA, Pinto A, Van Den Abbeele J, Ribeiro RM, Dias S, Smith TK, and Figueiredo LM (2016). Trypanosoma brucei parasites occupy and functionally adapt to the adipose tissue in mice. Cell Host Microbe 19(6): 837-848.

33. Grab DJ, Nikolskaia O, Kim YV, Lonsdale-Eccles JD, Ito S, Hara T, Fukuma T, Nyarko E, Kim KJ, Stins MF, Delannoy MJ, Rodgers J, and Kim KS (2004). African trypanosome interactions with an in vitro model of the human blood-brain barrier. J Parasitol 90(5): 970-979.
34. Giordani F, Morrison L, Rowan TG, DE Koning HP, and Barrett MP (2016). The animal trypanosomiases and their chemotherapy: a review. Parasitology 143(14): 1862-1889.

35. Magnus E, Vervoort T, and Van Meirvenne N (1978). A cardagglutination test with stained trypanosomes (C.A.T.T.) for the serological diagnosis of T. B. gambiense trypanosomiasis. Ann Soc Belg Med Trop 58(3): 169-176.

36. Coustou V, Guegan F, Plazolles N, and Baltz T (2010). Complete in vitro life cycle of Trypanosoma congolense: development of genetic tools. PLoS Negl Trop Dis 4(3): e618.

37. Florimond C, Sahin A, Vidilaseris K, Dong G, Landrein N, Dacheux D, Albisetti A, Byard EH, Bonhivers M, and Robinson DR (2015). BILBO1 is a scaffold protein of the flagellar pocket collar in the pathogen Trypanosoma brucei. PLoS Pathog 11(3): e1004654. 